\title{
Aortic pressure wave reflection in patients after successful aortic arch repair in early infancy
}

\author{
Tomoaki Murakami ${ }^{1,2}$, Atsuhito Takeda ${ }^{1}$, Hirokuni Yamazawa ${ }^{1}$, Shigeru Tateno ${ }^{2}$, \\ Yasutaka Kawasoe $^{2}$ and Koichiro Niwa ${ }^{2,3}$
}

Despite the apparently successful surgical repair of aortic coarctation, subsequent cardiovascular complications have sometimes been encountered. Aortic pressure wave reflection is one of the risk factors for developing cardiovascular diseases, and an enhancement of the pressure wave reflection has been reported in patients after aortic arch repair. To clarify this issue, the increase in pressure wave reflection was evaluated in patients $<15$ years old who underwent aortic arch repair. This study enrolled 35 patients after aortic arch repair in early infancy. All patients underwent cardiac catheterization, and in 20 patients, there was no pressure difference within the repaired aortic arch. The aortic pressure waveforms in patients after successful aortic arch repair were recorded using a pressure sensor-mounted catheter, and the augmentation index in the ascending aorta was calculated. The augmentation index in patients after an aortic arch repair was increased compared with control subjects, although there was no pressure difference between the ascending and descending aorta $(\boldsymbol{P}<0.0001)$. The increase in the augmentation index was correlated with the patient's age $(r=0.8932, P<0.0001)$ and with the left ventricular posterior wall thickness $(r=0.4075, P=0.0373)$. In patients who undergo aortic arch repair, the pressure wave reflection is accelerated, even when the aortic arch repair is 'successful'. This increase is one of the possible causes of left ventricular hypertrophy. Hypertension Research (2013) 36, 603-607; doi:10.1038/hr.2013.1; published online 14 February 2013

Keywords: aortic arch; aortic coarctation; hypertrophy; pressure wave reflection

\section{INTRODUCTION}

Aortic pressure augmentation caused by pressure wave reflection is a physiologically important phenomenon. It keeps the arterial blood flow constant while enhancing the coronary blood supply. However, excessive aortic pressure wave reflection contributes to an increase in cardiovascular diseases. ${ }^{1,2}$ To evaluate the degree of aortic pressure reflection, the augmentation index, which is obtained from the central arterial pressure waveform as the ratio of augmentation pressure to the total pulse pressure, is one of the most popular tools. ${ }^{3}$ In adults, significant evidence supports a correlation between the value of the augmentation index and the occurrence of cardiovascular diseases. ${ }^{4-6}$

Despite apparently successful aortic arch repair, defined as no residual pressure gradient after the surgical repair of aortic coarctation, subsequent cardiovascular complications have been encountered. ${ }^{7-12}$ One such complication is the early onset of cardiovascular diseases, such as hypertension, myocardial infarction, cardiac failure, stroke and sudden death. Problems have been reported to occur even when the aortic arch repair is successful. ${ }^{13-15}$

We previously reported the enhancement of the aortic pressure wave reflection in patients with aortic coarctation after repair. ${ }^{16}$ However, the study had two limitations. One was that some patients in the study had a small pressure gradient in their aortic arch, and the other was that the augmentation index of the patients was compared with age-matched, one-to-one controls in the report. The aortic augmentation index in children correlates with body height, not age. ${ }^{17}$ To resolve these problems and perform a quantitative analysis of enhanced pressure wave reflection, the augmentation index was calculated in patients who underwent aortic arch repair without a pressure gradient in their aortas and compared with reference data on the basis of height in this study. The purpose of this study was to prove the enhancement of aortic pressure wave reflection in patients after aortic arch repair was compared with normal patients on the basis of their height and quantify the increase in pressure wave reflection. Moreover, to clarify the influence of enhanced pressure wave reflection, the correlation between the increase in the pressure wave reflection and left ventricular wall thickness was also examined.

\section{PATIENTS AND METHODS}

Study subjects

In total, 35 patients who were $<15$ years old who had undergone an aortic arch repair for coarctation of the aorta (24 patients) or interrupted aortic arch (11 patients) in early infancy were enrolled. Although no patient had any subjective sign of cardiovascular dysfunction, cardiac catheterization proved small pressure differences between the ascending and descending aorta

${ }^{1}$ Department of Pediatrics, Hokkaido University, Graduate School of Medicine, Sapporo, Japan; ${ }^{2}$ Department of Adult Congenital Heart Disease and Pediatric Cardiology, Chiba Cardiovascular Center, Ichihara, Japan and ${ }^{3}$ Department of Cardiology, St Luke's International Hospital, Tokyo, Japan 
Table 1 Patients' characteristics $(n=20)$

\begin{tabular}{lc}
\hline Characteristics & Value \\
\hline Male/female & $14 / 6$ \\
Age (years) & $8.0 \pm 3.6$ \\
Height (cm) & $122.3 \pm 22.7$ \\
Weight (kg) & $26.1 \pm 13.3$ \\
Brachial SBP (mm Hg) & $107.3 \pm 15.6$ \\
$\quad$ Hypertension (+) & $2(10 \%)$ \\
Heart rate (b.p.m.) & $86.4 \pm 21.2$ \\
CoA/IAA & $14 / 6$ \\
Period after arch repair (years) & $7.9 \pm 3.6$ \\
VSD (+) & $14(70 \%)$ \\
Period after VSD repair (years) & $6.8 \pm 3.8$ \\
Arch repair method & \\
Extended end-to-end anastomosis & $13(65 \%)$ \\
Subclavian flap & $6(30 \%)$ \\
Blalock-Park & $1(5 \%)$
\end{tabular}

Abbreviations: CoA, coarctation of aorta; IAA, interruption of the aortic arch; SBP, systolic blood pressure; VSD, ventricular septal defect.

Values are mean \pm s.d. or number (\%)

$(<20 \mathrm{~mm} \mathrm{Hg})$ in 15 patients, and they were excluded from this study. Therefore, 20 patients (14 males and 6 females, 14 coarctation of the aorta and 6 interrupted aortic arch) aged 1-13 $(8.0 \pm 3.6)$ years were included in the analysis (Table 1). No patients had any significant leakage at the aortic level, including the patent ductus arteriosus and aortic regurgitation. The methods of aortic arch repair were extended end-to-end anastomosis in 13 patients, subclavian flap in 6 and Blalock-Park operation in 1. Aortic arch repair was performed at $24.0 \pm 23.7$ days after birth ( 3 days 3 months), and the period after aortic arch repair was $7.9 \pm 3.6$ years. Three patients had undergone balloon dilatation of the aortic arch after surgical arch repair. Fourteen patients had ventricular septal defect, which was closed at $0.8 \pm 0.5$ years old. Seven patients were diagnosed with a bicuspid aortic valve. None of them were taking any medication or diagnosed to be suffering from Turner syndrome. Their right brachial systolic blood pressure was $107.3 \pm 15.6 \mathrm{~mm} \mathrm{Hg}$, and the diastolic blood pressure was $59.1 \pm 15.6 \mathrm{~mm} \mathrm{Hg}$. Only two patients were diagnosed with hypertension based on Japanese guidelines for the management of hypertension (JSH 2009). One was diagnosed with systolic hypertension, and the other was diagnosed with systolic and diastolic hypertension. Their parents and siblings did not suffer from hypertension or diabetes. All subjects gave their written informed consent, and the Chiba Cardiovascular Center ethics committee approved the study protocol (No.945, 2011.12.16).

\section{Study protocol}

Left heart catheterization was performed by femoral arterial puncture. The ascending aortic pressure was measured using a pressure sensor-mounted catheter (model SPC-464D, Millar Instrument, Houston, TX, USA) before the injection of any contrast material. The ascending aortic pressure waveform was recorded at one vertebral body thickness higher than the aortic valve. The waveform was recorded on a hard disk through an analog-digital converter, and it was also simultaneously recorded with an electrocardiogram.

\section{Pulse wave analysis}

The systolic blood pressure, diastolic blood pressure and pulse pressure were measured in each patient from the recorded pressure waveforms. The inflection points or peaks that corresponded to early and late systolic blood pressure were obtained by multidimensional derivatives of the original pressure pulse waveforms. Using the pressure waveform, an augmentation index was calculated for each patient. ${ }^{18}$ The augmentation index is strongly influenced by heart rate. Therefore, the corrected augmentation index, which is equivalent to the augmentation index at a heart rate of 75 beats per minute, was also calculated using the following formula: ${ }^{19}$

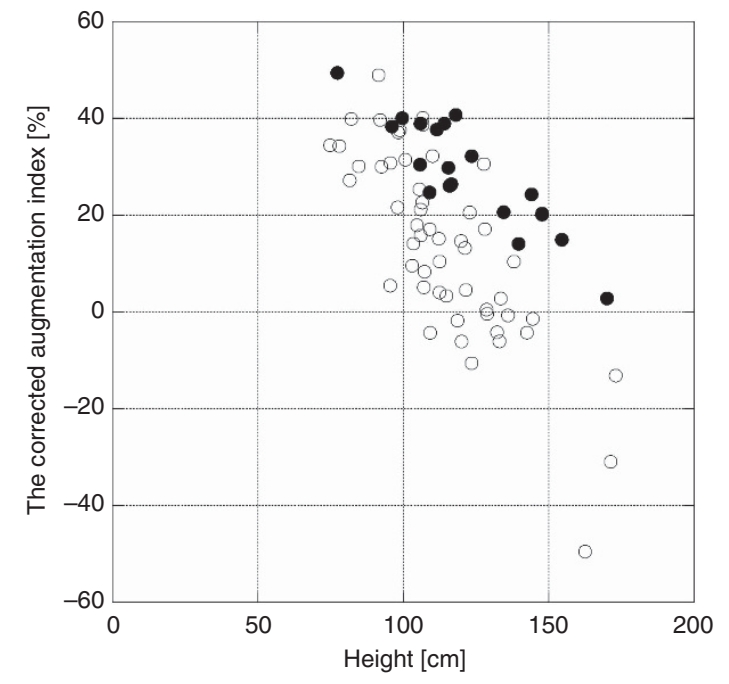

Figure 1 The relationship between the body height and the heart ratecorrected augmentation index of reference data (open circle) ${ }^{17}$ and in children $<15$ years of age after an aortic arch repair (closed circle). The index was significantly higher in the aortic arch repair group $(P<0.0001)$.

Corrected augmentation index $(\%)=$ augmentation index $(\%)+$ (heart rate (b.p.m.) -75$) \times 0.39$.

The corrected augmentation index in patients who underwent aortic arch repair was compared with reference data in children, which were analyzed in the same way. ${ }^{17}$

\section{Morphological analysis of the left ventricle}

To evaluate the degree of left ventricular hypertrophy, the end-diastolic left ventricular posterior wall thickness (LVPWTd) was measured using echocardiography. ${ }^{20}$ The value was converted to the percentage of normal LVPWTd (\%LVPWTd) using the known relationship between body surface area and LVPWTd in healthy Japanese children.

$\%$ LVPWTd $(\%)=\left(\operatorname{LVPWTd}(\mathrm{mm}) /\left(4.1 \times \log \left(\right.\right.\right.$ body surface area $\left.\left(\mathrm{m}^{2}\right)\right)$ $+6.1)) \times 100$.

\section{Statistical analysis}

Continuous values are expressed as the mean value \pm s.d. Categorical measures are presented as frequencies with percentages. The continuous variables were compared between groups using the Mann-Whitney test. The corrected augmentation index-body height relationship between the aortic arch repair group and the reference data was compared using analysis of covariance, with the corrected augmentation index as the dependent variable, arch repair as the factor and body height as the covariate. The strength of the correlation between the two groups was analyzed by Spearman's test. PRISM software version 4.0c (Graph Pad Software, La Jolla, CA, USA) was used for all statistical analyses without analysis of covariance, which was performed with SPSS (Statistical Package for the Social Sciences) software version 16.0 (SPSS, Chicago, IL, USA). A $P$ value $<0.05$ was considered to be statistically significant.

\section{RESULTS}

The systolic blood pressure in the ascending aorta was $100.8 \pm 10.4 \mathrm{~mm} \mathrm{Hg}$, and the diastolic blood pressure was $61.8 \pm 8.2 \mathrm{~mm} \mathrm{Hg}$. The augmentation index was $24.1 \pm 10.4 \%$, and the corrected augmentation index was $28.5 \pm 11.3 \%$. Figure 1 shows the relationship between the corrected augmentation index and body height in patients who underwent aortic arch repair in comparison with the reference data. The corrected augmentation index was significantly higher in patients after aortic arch repair than the reference data $(P<0.0001)$. The increase in the index compared with 


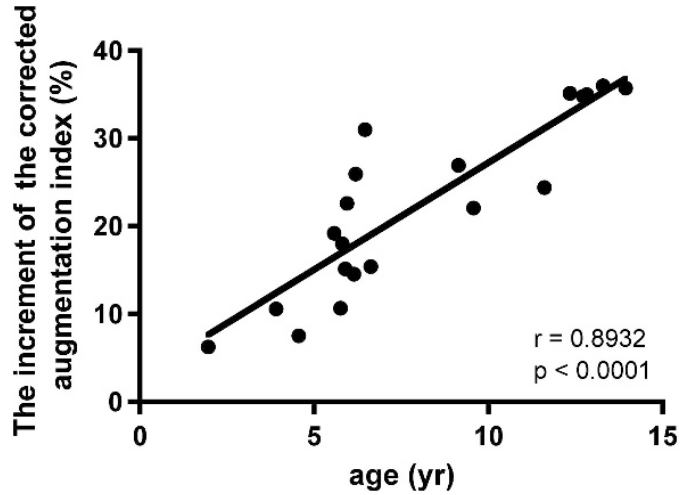

Figure 2 The relationship between the age and the increment of the corrected augmentation index in patients after the repair of aortic arch. The increment increased with age $(P<0.0001)$.

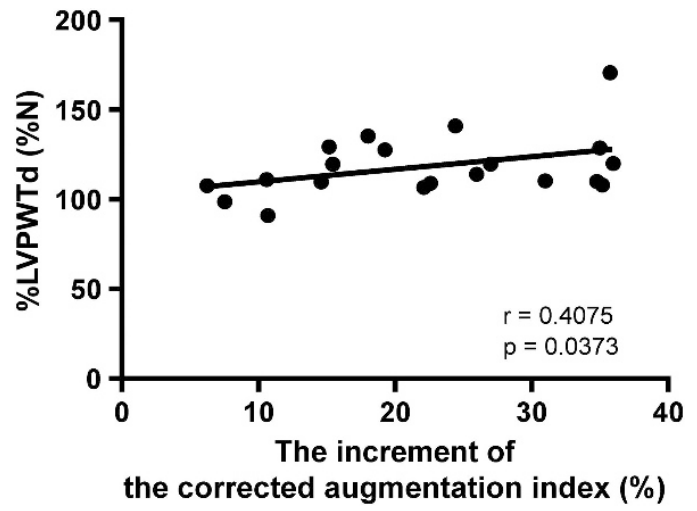

Figure 3 The relationship between the increment of the corrected augmentation index and the percentage of the end-diastolic left ventricular posterior wall thickness (\%LVPWTd) in patients after the repair of aortic arch. The posterior wall thickness had weak positive correlation with the increment of the corrected augmentation index $(r=0.4075, P=0.0373)$

the estimated value based on the body height was $22.3 \pm 10.0 \%$, and there was a positive correlation between the increase in the index and the patient age (Figure $2 ; r=0.8932, P<0.0001$ ). The \%LVPWTd of the patients was $118.5 \pm 17.4 \%$. The \%LVPWTd had a positive relationship with the increase in the index (Figure 3; $r=0.4075$, $P=0.0373$ ).

The basic diseases (coarctation of the aorta vs. interrupted aortic arch) and the type of repair method (extended end-to-end anastomosis vs. subclavian flap) did not have an effect on the increase in the corrected augmentation index or \%LVPWTd (Tables 2 and 3). The increase in the corrected augmentation index and \%LVPWTd was also not influenced by the presence of a bicuspid aortic valve (Table 4).

\section{DISCUSSION}

This report demonstrates enhanced aortic pressure wave reflection in patients after aortic arch repair in early infancy, even without pressure differences in the aortic arch (Figure 1), and the enhancement of the pressure wave reflection increased with the patient's age (Figure 2). Moreover, the enhancement was significantly correlated with left ventricular hypertrophy.

We previously demonstrated that the enhancement of pressure wave reflection after aortic arch repair originated from the reconstructed portion. ${ }^{16,21}$ In normal aortic circulation, major pressure
Table 2 Basic diseases of the aorta

\begin{tabular}{lccc}
\hline & CoA $(\mathrm{n}=14)$ & $I A A(\mathrm{n}=6)$ & $\mathrm{P}$ \\
\hline Male/female & $10 / 4$ & $4 / 2$ & \\
Age (years) & $7.4 \pm 3.5$ & $9.4 \pm 3.8$ & $\mathrm{NS}$ \\
Height (cm) & $119.1 \pm 23.3$ & $129.9 \pm 21.2$ & $\mathrm{NS}$ \\
Weight (kg) & $25.0 \pm 14.4$ & $28.6 \pm 10.9$ & $\mathrm{NS}$ \\
Brachial SBP (mm Hg) & $106.2 \pm 18.5$ & $109.7 \pm 7.8$ & $\mathrm{NS}$ \\
Period after arch repair (years) & $7.3 \pm 3.5$ & $9.4 \pm 3.8$ & $\mathrm{NS}$ \\
Arch repair method & & & \\
$\quad$ Extended end-to-end anastomosis & $8(57 \%)$ & $5(83 \%)$ & \\
Subclavian flap & $6(43 \%)$ & $0(0 \%)$ & \\
$\quad$ Blalock-Park & $0(0 \%)$ & $1(17 \%)$ & \\
dAI & $29.8 \pm 12.5$ & $25.7 \pm 11.3$ & $\mathrm{NS}$ \\
\%LVPWTd & $121.7 \pm 18.5$ & $111.2 \pm 12.8$ & $\mathrm{NS}$ \\
\hline
\end{tabular}

Abbreviations: CoA, coarctation of the aorta; dAl, increment of corrected augmentation index; IAA, interrupted aortic arch; \%LVPWTd, percentage of the end-diastolic left ventricular posterior wall thickness; NS, not significant; SBP, systolic blood pressure.

Values are mean \pm s.d. or number (\%).

Table 3 Repair methods of aortic arch

\begin{tabular}{lccc}
\hline & EEEA $(\mathrm{n}=13)$ & SCF $(\mathrm{n}=6)$ & $\mathrm{P}$ \\
\hline Male/female & $8 / 5$ & $5 / 1$ & \\
Age (years) & $6.9 \pm 3.2$ & $9.5 \pm 3.7$ & $\mathrm{NS}$ \\
Height (cm) & $116.2 \pm 21.3$ & $132.1 \pm 23.7$ & $\mathrm{NS}$ \\
Weight (kg) & $22.8 \pm 9.9$ & $32.3 \pm 18.8$ & $\mathrm{NS}$ \\
Brachial SBP (mm Hg) & $107.8 \pm 10.7$ & $106.3 \pm 24.3$ & NS \\
Period after arch repair (years) & $6.8 \pm 3.2$ & $9.4 \pm 3.6$ & NS \\
Basic diseases & & & \\
$\quad$ Coarctation of aorta & $8(62 \%)$ & $6(100 \%)$ & \\
$\quad$ Interruption of the aortic arch & $5(38 \%)$ & $0(0 \%)$ & \\
dAI & $19.3 \pm 9.7$ & $26.8 \pm 8.4$ & NS \\
\%LVPWTd & $113.1 \pm 12.4$ & $130.0 \pm 23.3$ & NS \\
\hline Abbrevians: & & &
\end{tabular}

Abbreviations: dAI, increment of corrected augmentation index; EEEA, extended end-to-end anastomosis of aortic arch; \%LVPWTd, the percentage of the end-diastolic left ventricular posterior wall thickness; NS, not significant; SBP, systolic blood pressure; SCF, subclavian flap. Values are mean \pm s.d. or number (\%).

wave reflection occurs in the region of the main aortic bifurcation. As the patients grow, the distance between the reconstructed portion and the main aortic bifurcation becomes longer. Therefore, the difference in the aortic pressure augmentation between the repaired aorta group and the normal aortic circulation group should tend to increase as they grow. The difference in the corrected augmentation index is approximately $40 \%$ when the patients attain their peak body height. Although the mid-teens is the period in which the index is the lowest, ${ }^{17}$ the value in mid-teen patients who undergo aortic arch repair approximately corresponds to that of a middle-aged person with a normal aorta. ${ }^{3}$ Moreover, our data suggested that the index in patients was positive throughout their life. The positive augmentation index indicates that the reflection wave returns to the heart before aortic valve closure. Therefore, it leads to an increase in the afterload in the left ventricle.

The patients' left ventricular wall was thick, and the degree of thickness was correlated with the increase in pressure wave reflection (Figure 3). Left ventricular hypertrophy occurs even after the repair of aortic coarctation. ${ }^{22}$ Because the enhanced aortic pressure wave reflection returns to the heart when the aortic valve is open, it leads to an increase in afterload for the left ventricle and can cause left 
Table 4 Influences of bicuspid aortic valve

\begin{tabular}{lccc}
\hline & BAV $(-)(\mathrm{n}=13)$ & BAV $(+)(\mathrm{n}=7)$ & $\mathrm{P}$ \\
\hline Male/female & $9 / 4$ & $5 / 2$ & \\
Age (years) & $7.8 \pm 3.9$ & $8.4 \pm 3.2$ & NS \\
Height (cm) & $121.2 \pm 25.9$ & $124.4 \pm 16.7$ & NS \\
Weight (kg) & $27.0 \pm 15.4$ & $24.4 \pm 9.1$ & NS \\
Brachial SBP (mm Hg) & $106.5 \pm 18.4$ & $108.7 \pm 10.8$ & NS \\
Period after arch repair (years) & $7.7 \pm 3.9$ & $8.3 \pm 3.2$ & NS \\
Basic diseases & & & \\
$\quad$ Coarctation of aorta & $9(69 \%)$ & $5(71 \%)$ & \\
$\quad$ Interruption of the aortic arch & $4(31 \%)$ & $2(29 \%)$ & \\
dAI & $21.6 \pm 10.6$ & $23.8 \pm 9.5$ & NS \\
\%LVPWTd & $119.3 \pm 19.3$ & $117.0 \pm 14.3$ & NS \\
\hline
\end{tabular}

Abbreviations: BAV, bicuspid aortic valve, dAl, increment of corrected augmentation index, EEEA, extended end-to-end anastomosis of aortic arch, \%LVPWTd, the percentage of the enddiastolic left ventricular posterior wall thickness, NS, not significant; SBP, systolic blood pressure, SCF, subclavian flap.

Values are mean \pm s.d. or number $(\%)$ workload of the left ventricle and make the ventricle potentially more vulnerable to any number of heart diseases, especially ischemic heart disease. Many reports have demonstrated the relationship between increased pressure wave reflection and cardiovascular diseases. ${ }^{1,2}$ Moreover, future cardiovascular diseases are reported to be one of the most important problems in patients with aortic coarctation after repair. ${ }^{7,12,13,15}$ Enhanced aortic pressure wave reflection could be one of the causes of future cardiovascular diseases.

In the present data, only two patients demonstrated hypertension in childhood. It has been reported the blood pressure is normal in most aortic arch-repaired patients 5-10 years after surgery, but the incidence of hypertension in patients increases over time. Clarkson et al. ${ }^{23}$ reported that the incidence of normal blood pressure in patients was $69 \%$ at 10 years, $55 \%$ at 15 years and $20 \%$ at 25 years postoperatively. The hypertension in patients who undergo aortic arch repair is progressive. We previously reported elevated central systolic blood pressure and pulse pressure in pediatric patients after aortic arch repair compared with age-matched controls. ${ }^{16}$ Those factors are responsible for the development of left ventricular hypertrophy $^{24}$ and atherosclerosis. ${ }^{25}$ Niwa et al. ${ }^{26}$ concluded that the histological abnormality of aortas in patients with aortic coarctation was inherently pathogenic. Enhanced aortic pressure wave reflection, which remains even after repair of the aortic arch, should accelerate the congenital aortic structural abnormality. 27,28

Our data demonstrated that aortic arch repair itself was one of the risks of future cardiovascular diseases. The procedure results in the discontinuity of elasticity in the aorta, and it could become the point of pressure wave reflection. To prevent future cardiovascular diseases, patients should be educated concerning the cardiovascular risk from their early life and prevent the accumulation of other cardiovascular risk factors. Some types of drugs, including nitrates, angiotensinconverting enzyme inhibitors and calcium antagonists, are known to reduce pressure wave reflection in normal circulation. ${ }^{29}$ It should be examined whether such drugs are able to improve the prognosis of patients after aortic arch repair.

In our data, the basic diseases of the aorta, the type of aortic arch repair and the existence of a bicuspid aortic valve did not influence the increase in pressure wave reflection. However, our results, especially subgroup analyses, were demonstrated in a small number of patients. Angiography of the repaired aorta demonstrated that a normal aortic shape was achieved when the aorta was repaired by extended end-to-end anastomosis rather than being repaired by the subclavian flap method. Table 3 demonstrates that the increase in the corrected augmentation index and the \%LVPWTd in patients repaired with the subclavian flap method was higher than in patients repaired with extended end-to-end anastomosis, although the difference was not statistically significant. To further investigate this subgroup, it might be necessary to examine more patients, and recent advances in an instrument that can non-invasively evaluate aortic circulation will make this possible.

In conclusion, even after successful aortic arch repair, the aortic pressure wave reflection increased in patients with a repaired aorta, and it contributed to left ventricular hypertrophy. This increase could be one of the causes of future hypertension and cardiovascular diseases.

\section{CONFLICT OF INTEREST}

The authors declare no conflict of interest.

1 O'Rourke MF. Arterial stiffness, systolic blood pressure, and logical treatment of arteria hypertension. Hypertension 1990; 15: 339-347.

2 Nichols WW, Edwards DG. Arterial elastance and wave reflection augmentation of systolic blood pressure: deleterious effects and implications for therapy. J Cardiovasc Pharmacol Ther 2001; 6: 5-21.

3 Wave reflections. In Nichols WW and O'Rourke MF (eds), Mcdonald's blood flow in arteries. Hodder Arnold: London, 193-214, 2005.

4 Weber T, Auer J, O'Rourke MF, Kvas E, Lassnig E, Berent R, Eber B. Arterial stiffness, wave reflections, and the risk of coronary artery disease. Circulation 2004; 109 . 184-189.

5 Kingwell BA, Waddell TK, Medley TL, Cameron JD, Dart AM. Large artery stiffness predicts ischemic threshold in patients with coronary artery disease. J Am Coll Cardiol 2002; 40: 773-779.

6 Hayashi T, Nakayama Y, Tsumura K, Yoshimaru K, Ueda H. Reflection in the arteria system and the risk of coronary heart disease. Am J Hypertens 2002; 15: 405-409.

7 Daniels SR. Repair of coarctation of the aorta and hypertension: does age matter? Lancet 2001; 358: 89.

8 Kaplan S, Perloff JK. Survival patterns after cardiac surgery or interventiona catheterization. In Perloff JK and Child JS (eds), Congenital Heart Disease in Adults. WB Saunders: Philadelphia, 54-87, 1998.

9 Presbitero P, Demarie D, Villani M, Perinetto EA, Riva G, Orazan F, Bobbio M, Morea M, Brusca A. Long term results (15-30 years) of surgical repair of aortic coarctation. $\mathrm{Br}$ Heart J 1987; 57: 462-467.

10 Stewart AB, Ahmed R, Travill CM, Newman CG. Coarctation of the aorta life and health 20-44 years after surgical repair. Br Heart J 1993; 69: 65-70.

11 Jenkins NP, Ward C. Coarctation of the aorta: natural history and outcome after surgical treatment. $Q$ J Med 1999; 92: 365-371.

12 Cohen M, Fuster V, Steele PM, Driscoll D, McGoon DC. Coarctation of the aorta: long-term follow-up and prediction of outcome after surgical correction. Circulation 1989; 80: 840-845.

13 Celermajer DS, Greaves K. Survivors of coarctation repair: fixed but not cured. Heart 2002; 88: 113-114.

14 Toro-Salazar OH, Steinberger J, Thomas W, Rocchini AP, Carpenter B, Moller JH. Longterm follow-up patients after coarctation of the aorta repair. Am J Cardiol 2002; 89: 541-547.

15 O'Sullivan JJ, Derrick G, Darnell R. Prevalence of hypertension in children after early repair of coarctation of the aorta: a cohort study using casual and $24 \mathrm{~h}$ blood pressure measurement. Heart 2002; 88: 163-166.

16 Murakami T, Takeda A. Enhanced aortic pressure wave reflection in patients after repair of aortic coarctation. Ann Thorac Surg 2005; 80: 995-999.

17 Murakami T, Takeda A, Takei K, Ueno M, Yakuwa S, Yamazawa H, Furukawa T. Aortic pressure wave reflection in children. Hypertens Res 2010; 33: 225-228.

18 Murgo JP, Westerhof N, Giolma JP, Altobelli SA. Aortic input impedance in norma man: relationship to pressure waveforms. Circulation 1980; 62: 105-116.

19 Wilkinson IB, MacCallum H, Flint L, Cockcroft JR, Newby DE, Webb DJ. The influence of heart rate on augmentation index and central arterial pressure in humans. $J$ Physiol 2000; 525.1: 263-270.

20 Sahn DJ, DeMaria A, Kisslo J, Weyman A. Echocardiography the Committee on MMode Standardization of the American Society of Echocardiography. Recommendations regarding quantitation in m-mode echocardiography: results of a survey of echocardiographic measurements. Circulation 1978; 58: 1072-1083.

21 Murakami T, Ueno M, Takeda A, Yakuwa S. Pressure wave reflection after successful balloon dilatation of aortic coarctation. Circ J 2007; 71: 1821-1822. 
22 de Divitiis M, Pilla C, Kattenhorn M, Donald A, Zadinello M, Wallace S, Redington A, Deanfield J. Ambulatory blood pressure, left ventricular mass, and conduit artery function late after successful repair of coarctation of the aorta. J Am Coll Cardiol 2003; 41: 2259-2265.

23 Clarkson PM, Nicholson MR, Barratt-Boyes BG, Neutze JM, Whitlock RM. Results after repair of coarctation of the aorta beyond infancy: a 10-28 year follow-up with particular reference to late systemic hypertension. Am J Cardiol 1983; 51: 1481-1488.

24 Hundley WG, Kitzman DW, Morgan TM, Hamilton CA, Darty SN, Stewart KP, Herrington DM, Link KM, Little WC. Cardiac cycle-dependent changes in aortic area and distensibility are reduced in older patients with isolated diastolic heart failure and correlate with exercise intolerance. J Am Coll Cardiol 2001; 38: 796-802.

25 Nissen SE, Tuzcu EM, Libby P, Thompson PD, Ghali M, Garza D, Berman L, Shi H, Buebendorf E, Topol EJ. Effect of antihypertensive agents on cardiovascular events in patients with coronary disease and normal blood pressure. J Am Med Assoc 2004; 292: 2217-2226.

26 Niwa K, Perloff JK, Bhuta SM, Laks H, Drinkwater DC, Child JS, Miner PD. Structural abnormalities of great arterial walls in congenital heart disease. Circulation 2001; 103 : 393-400.

27 Isner JM, Donaldson RF, Fulson D, Bhan I, Payne DD, Cleveland RJ. Cystic medial necrosis in coarctation of the aorta: A potential factor contributing to adverse consequences observed after percutaneous balloon angioplasty of coarctation sites. Circulation 1987; 75: 689-695.

28 Zile MR, Isner JM, Bing OHL, Gaasch WH. Cystic medial necrosis in the ascending aorta of puppies with experimental ascending aortic coarctation. J Am Coll Cardiol 1985; 5: 488.

29 O'Rourke MF. Arterial stiffening and vascular/ventricular interaction. J Hum Hypertens 1994; 8 (Suppl 1): S9-S15. 